\title{
Magnetic Moment and Anisotropy of Individual Co Atoms on Graphene
}

\author{
F. Donati, ${ }^{1}$ Q. Dubout, ${ }^{1}$ G. Autès, ${ }^{2}$ F. Patthey, ${ }^{1}$ F. Calleja, ${ }^{1,3}$ P. Gambardella, ${ }^{1,4,5}$ O. V. Yazyev, ${ }^{2}$ and H. Brune ${ }^{1, *}$ \\ ${ }^{1}$ Institute of Condensed Matter Physics (ICMP), Ecole Polytechnique Fédérale de Lausanne (EPFL), Station 3, CH-1015, Switzerland \\ ${ }^{2}$ Institute of Theoretical Physics, Ecole Polytechnique Fédérale de Lausanne (EPFL), CH-1015 Lausanne, Switzerland \\ ${ }^{3}$ Madrid Institute for Advanced Studies, IMDEA Nanoscience, Calle Faraday 9, Campus Cantoblanco, E-28049 Madrid, Spain \\ ${ }^{4}$ Catalan Institute of Nanoscience and Nanotechnology (ICN2), UAB Campus, E-08193 Barcelona, Spain \\ ${ }^{5}$ Department of Materials, ETH Zurich, CH-8093 Zurich, Switzerland
}

(Received 14 August 2013; published 3 December 2013)

\begin{abstract}
We report on the magnetic properties of single Co atoms on graphene on $\operatorname{Pt}(111)$. By means of scanning tunneling microscopy spin-excitation spectroscopy, we infer a magnetic anisotropy of $K=-8.1 \mathrm{meV}$ with out-of-plane hard axis and a magnetic moment of $2.2 \mu_{B}$. Co adsorbs on the sixfold graphene hollow site. Upon hydrogen adsorption, three differently hydrogenated species are identified. Their magnetic properties are very different from those of clean Co. Ab initio calculations support our results and reveal that the large magnetic anisotropy stems from strong ligand field effects due to the interaction between Co and graphene orbitals.
\end{abstract}

DOI: 10.1103/PhysRevLett.111.236801

PACS numbers: 73.22.Pr, 32.10.Dk, 75.30.Gw, 75.70.Rf

Graphene is a promising material for spintronics due to the possibility of realizing controllable spin transport [1], its perfect spin filtering [2], and spin-relaxation lengths of several micrometers at room temperature [3]. Doping graphene by magnetic impurities opens further possibilities [4-6]. In particular, the creation of extended magnetic phases [7], quantum critical Kondo anomalies [8,9], and the strong scattering of spin currents [10] have been predicted. Moreover, for $3 d$ metal atoms on graphene, the calculated uniaxial magnetic anisotropies [11,12] are beyond the current record value for a surface-adsorbed atom [13]. Furthermore, hydrogen adsorption has been predicted to change the spin of the adatoms [14], underlining its potential to tailor the magnetic properties. However, the predicted anisotropies and moments are highly controversial and largely depend on how electron correlations are treated. For the prototypical system of Co atoms on graphene, spin moments between 1 and $3 \mu_{B}$ and anisotropies of different signs have been calculated [12,15-17]. At present, there is only one experiment addressing the magnetic properties of transition-metal adatoms on graphene. It reports on-top adsorption, a high-spin ground state, and weak magnetic anisotropy for $\mathrm{Co} /$ graphene/SiC(0001) [18].

Here we present the first local measurement of the magnetic moment and anisotropy of individual Co atoms on graphene on $\mathrm{Pt}(111)$. This substrate was chosen since graphene binds very weakly to it [19], thus approaching freestanding graphene. Using scanning tunneling microscopy (STM) spin-excitation spectroscopy [20], we find an exceptionally large magnetic anisotropy of $K=-8.1 \pm$ $0.4 \mathrm{meV}$ with out-of-plane hard axis and a magnetic moment of $2.2 \pm 0.4 \mu_{B}$. Fully relativistic density functional theory (DFT) calculations show the anisotropy to be mainly a hybridization effect. In addition to clean Co, we identify three hydrogenated species, $\mathrm{CoH}_{n}, n \in\{1,2,3\}$, with very different magnetic behavior. The coexistence of clean and hydrogenated adatoms is expected to be a general feature of transition metal atoms on graphene that has to be considered in the interpretation of any ensemble measurement.

The experiments were performed with a homebuilt STM operating at $T=0.4 \mathrm{~K}$ and in magnetic fields up to $B=8.5 \mathrm{~T}$ perpendicular to the surface. $\mathrm{Pt}(111)$ was prepared by means of $\mathrm{Ar}^{+}$sputtering and flash annealing $(1400 \mathrm{~K})$ cycles until surface impurity concentrations $<0.5 \%$ were reached. Graphene patches of various sizes $(50-500 \mathrm{~nm})$ were grown by both $\mathrm{C}$ segregation $(1500 \mathrm{~K}$, 1-3 min) and chemical vapor deposition $\left(10 \mathrm{~L} \mathrm{C}_{2} \mathrm{H}_{4}\right.$ at $1230 \mathrm{~K}) .5 .0 \pm 0.5 \times 10^{-3}$ monolayers of Co were deposited in situ at $16 \mathrm{~K}$ and $p_{\text {tot }} \leq 8 \times 10^{-10}$ mbar using an $e$-beam evaporator with a $99.995 \%$ purity Co rod. This led to individual immobile adatoms [21]. Constant-current STM images were acquired with a W tip at tunnel current $I_{t}$ and sample bias $V_{t}$ given in the figure captions. $d I / d V$ spectra were recorded with open feedback and lock-in using the indicated peak-to-peak modulation voltage $V_{\text {mod }}$ at frequency $f . d^{2} I / d V^{2}$ spectra were obtained by numerical differentiation.

The first-principles DFT calculations used the local density approximation with a mean-field Hubbard correction $(\mathrm{LDA}+U)[22,23]$, as implemented in QUANTUMESPRESSO [24]. We chose $U=4 \mathrm{eV}$ for Co in agreement with previous calculations [15]. Spin-orbit effects were accounted for by using the fully relativistic norm-conserving pseudopotentials acting on valence electron wave functions represented in the two-component spinor form [25]. The $\mathrm{CoH}_{n}, n \in\{0,1,2,3\}$, adsorption complexes were placed in a $(4 \times 4)$ supercell of graphene and the structures were fully relaxed. The magnetic anisotropy energy was obtained from the difference of total energies of the out-of-plane and 
in-plane spin configurations. The first-principles simulated STM images show the tunnel current at a constant height of $z=4 \AA$ above the Co adatom and use the Tersoff-Hamann approximation [26]. The atomic spin and orbital moments, as well as the electronic configuration of the Co adatom, were obtained by projecting the wave functions of the total system onto atomic orbitals of the Co atom.

Figure 1(a) shows an atomically resolved image of the graphene layer. It appears as a hexagonal lattice of protrusions, one per graphene unit cell [27,28]. This can be rationalized by the very small difference in substrate binding energy of the two $\mathrm{C}$ atoms in the graphene unit cell. Hence, the contrast is dominated by the difference between $\mathrm{C}_{6}$ rings and sixfold hollow sites. The latter are imaged as protrusions with the present tip and tunnel parameters. In addition, one distinguishes the graphene moire pattern caused by the misfit with the $\operatorname{Pt}(111)$ substrate [27,29,30]. Graphene on $\operatorname{Pt}(111)$ exhibits a variety of moiré structures [27,30]. The results presented here were obtained on the one with a period of $(4 \times 4)$ graphene unit cells; however, they are characteristic of Co adatoms also on the other moiré structures.

A STM image of three Co monomers adsorbed on graphene is shown in Fig. 1(b). The adatoms appear as
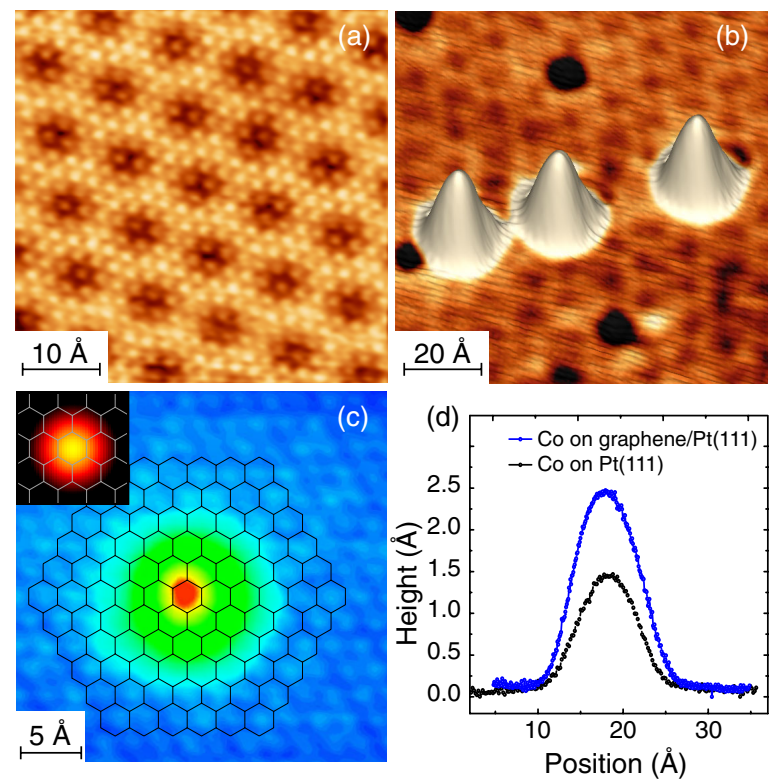

FIG. 1 (color online). (a) STM image of graphene/Pt(111) showing atomic contrast together with moiré pattern $\left(V_{t}=\right.$ $-1 \mathrm{mV}, I_{t}=8 \mathrm{nA}, T=4.5 \mathrm{~K}$ ). (b) STM image of three Co adatoms $\left(V_{t}=-20 \mathrm{mV}, I_{t}=1 \mathrm{nA}, T=1.7 \mathrm{~K}\right)$. (c) Hollow adsorption site inferred from adsorbate apex with respect to atomically resolved $\mathrm{C}$ lattice sketched in black $\left(V_{t}=\right.$ $\left.-50 \mathrm{mV}, I_{t}=100 \mathrm{pA}, T=4.5 \mathrm{~K}\right)$. Inset: Simulated STM image. (d) Line profile of Co atom on graphene/Pt(111) (blue dots, $\mathrm{FWHM}=9.0 \AA$ ) and on $\operatorname{Pt}(111)$ (black dots, FWHM = 8.3 $\AA$ ) taken with the same tip $\left(V_{t}=-100 \mathrm{mV}, I_{t}=100 \mathrm{pA}\right.$, $T=4.5 \mathrm{~K})$.

$2.4 \AA$ high protrusions. The long-range corrugation of the moiré pattern is also visible. A close-up of one Co adatom is shown together with the atomically resolved graphene lattice in Fig. 1(c). From the extrapolation of the graphene lattice close to the adsorbate, we conclude that its apex is located on the sixfold hollow site. This adsorption site corresponds to the lowest energy configuration in our calculations, and the simulated STM image shown in the inset agrees very well with the experiment. The lateral extent of the adsorbate on graphene is, after taking the different apparent height into account, identical to the one on $\operatorname{Pt}(111)$, as shown in Fig. 1(d). Therefore, the adsorbate and the electron charge are very well localized. The same adsorption site was also reported for $\mathrm{Co}$ atoms on H-intercalated graphene/SiC(0001) [31]. For that system, hollow and top site adsorption coexist, while only top site was observed for graphene/SiC(0001) [18].

After evaporating Co onto graphene, we distinguish four Co-related adsorption species by their apparent heights and inelastic conductance steps, as shown in Figs. 2(a)-2(c). We label them $A, B, C$, and $D$, in order of increasing abundance on a freshly prepared sample. The differential conductance features of $B$ and $C$ show no Zeeman splitting in an external field [32] and are therefore very likely of vibrational origin, very similar to the ones reported for $\mathrm{Co} /$ graphene $/ \mathrm{SiO}_{2} / \mathrm{Si}(100)$ [5] and for hydrogenated transition metal and rare earth atoms on $\operatorname{Ag}(100)$, where they have been attributed to frustrated translations [33]. In contrast, and as we will see in detail below, the conductance steps characterizing adsorbates $A$ and $D$ show a clear Zeeman splitting proving their magnetic origin [20].
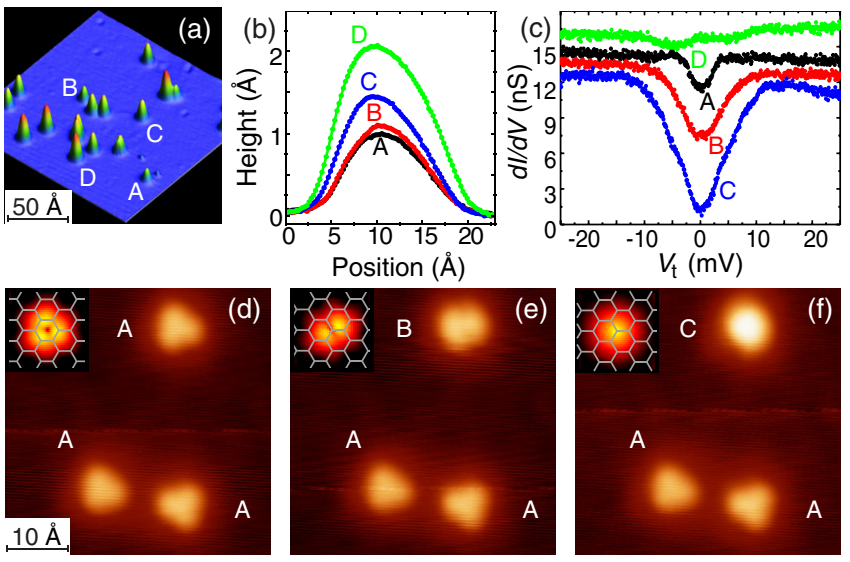

FIG. 2 (color online). (a),(b) Distinct apparent heights for clean $\mathrm{Co}(D), \mathrm{CoH}(C), \mathrm{CoH}_{2}(B)$, and $\mathrm{CoH}_{3}(A)\left(V_{t}=\right.$ $-25 \mathrm{mV}, I_{t}=50 \mathrm{pA}, T=4.5 \mathrm{~K}$ ). (c) Characteristic inelastic conductance steps of the four species (feedback gated at $V_{t}=-25 \mathrm{mV}$ and $I_{t}=250 \mathrm{pA}, V_{\text {mod }}=1 \mathrm{mV}, f=611 \mathrm{~Hz}$, $T=4.5 \mathrm{~K}$ ). $A, B$, and $D$ spectra are offset for clarity. (d) Three $\mathrm{CoH}_{3}$ complexes imaged as triangles. (e),(f) Successive dehydrogenation of the upper one by voltage pulses $\left(V_{t}=-100 \mathrm{mV}\right.$, $I_{t}=50 \mathrm{pA}, T=0.4 \mathrm{~K}$ ). Insets: Simulated STM images of the corresponding $\mathrm{CoH}_{n}$ complexes. 
In order to identify the four species, we note that adsorption complex $A$ can be transformed into $B$ by applying a tunnel voltage of $\left|V_{t}\right|=155 \pm 11 \mathrm{mV}$ with the tip positioned above the adsorbate. This transformation can be continued towards type $C$ with $\left|V_{t}\right|=180 \pm 7 \mathrm{mV}$, and this type is finally converted into type $D$ with $\left|V_{t}\right|=454 \pm$ $31 \mathrm{mV}$ [32]. Further increase of the tunnel voltage leads to a jump of the adsorbate to another hollow site, without altering its type $D$ character. The irreversibility of this transformation suggests a successive chemical modification of the adsorbed species that is terminated once type $D$ is reached.

Individual adsorbed transition metal atoms have been proven to dissociate $\mathrm{H}_{2}$ with very high efficiency [34]. We observe that direct exposure of the sample to $\mathrm{H}_{2}$ transforms all species almost entirely into type $A$. Similarly, exposure to the residual gas of the UHV chamber surrounding the cryostat augments the abundance of $A$ while $D$ strongly decreases [32]. This identifies type $A$ as most and type $D$ as least hydrogenated one. A comparison of high-resolution STM images with simulated ones identifies the intermediate species. Figure 2(d) shows that type $A$ has a triangular envelope in striking agreement with the simulated STM image of $\mathrm{CoH}_{3}$ shown in the inset. The upper adsorbate in Figs. 2(d)-2(f) has successively been transformed with voltage pulses as described above, while the lower two adsorbates remained unchanged in nature and appearance, proving that the tip apex did not change. Type $B$ is imaged as two distinct protrusions, while $C$ is imaged as a single one, both in excellent agreement with the simulated STM images for $\mathrm{CoH}_{2}$ and $\mathrm{CoH}$; see insets. Altogether, these observations unequivocally identify species $D$ as clean Co and $C, B, A$ as $\mathrm{CoH}_{n}, n \in\{1,2,3\}$. The three adsorbates in Fig. 1(b) are of type $D$; i.e., they are clean Co adatoms.
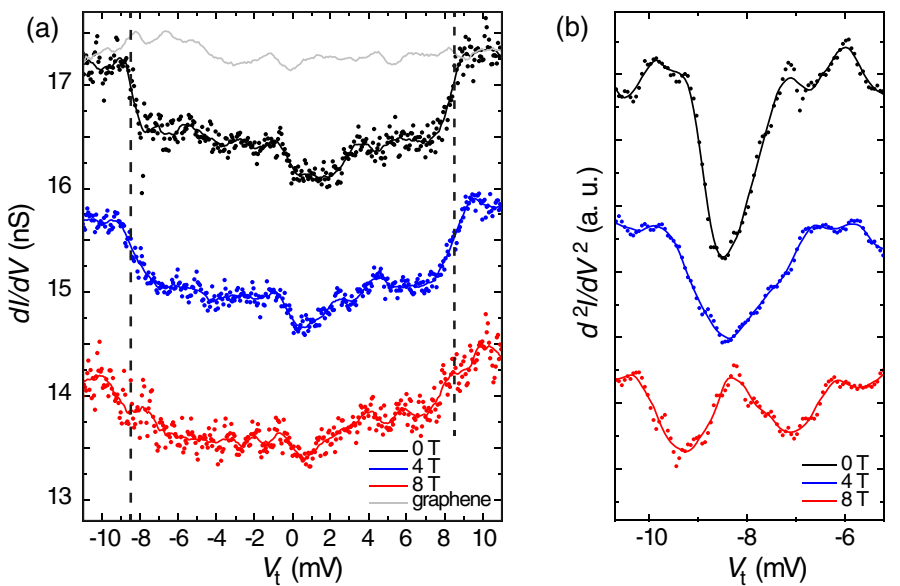

We now focus on the magnetic properties of the clean species. Their differential conductance shown in Fig. 3(a) is dominated by two steps at $\pm 8.1 \mathrm{mV}$. The step width at $B=0 \mathrm{~T}$ is with $\Delta E=950 \pm 350 \mu \mathrm{eV}$, after correction for modulation and $T$ broadening, comparable to reported values for single atoms on metal surfaces [35]. The smaller features visible at -0.5 and $2.5 \mathrm{meV}$ are not reproducible with different tips and therefore stem from the tip. The two steps progressively split in increasing out-of-plane magnetic fields, which is best seen in the $d^{2} I / d V^{2}$ spectra shown in Fig. 3(b).

The field dependence of the step energies $E_{\text {step }}$ is presented in Fig. 3(c). The single step splits into two with equal amplitude [see Fig. 3(b)], implying a transition between a singlet ground state $(m=0)$ and a doublet excited state $(m= \pm 1)$, and thus an integer value for $S$ [see Fig. 3(d)]. As a general tendency, the occupation of the Co $d$ states increases upon adsorption on a conducting substrate and values of $S \leq 3 / 2$ are normally observed [36]. Therefore, $S=1$ is the most reasonable spin multiplicity accounting for our findings. This value is supported by our first-principles calculations [see Fig. 3(e)] and consistent with other theoretical predictions for Co atoms on graphene [15].

To access the magnetic anisotropy, the field dependence of the step energies has been modeled by the following spin Hamiltonian [37]:

$$
\hat{H}_{\text {spin }}=g \mu_{\mathrm{B}} \hat{\mathbf{S}} \cdot \mathbf{B}+D \hat{S}_{z}^{2}
$$

where $g$ is the electron Landé factor, $\mathbf{B}$ denotes the external magnetic field, $D$ the uniaxial anisotropy parameter, and $\hat{S}$ the adatom total spin operator [38,39]. The $z$ axis is chosen by convention such as to maximize $|D|$. For the present
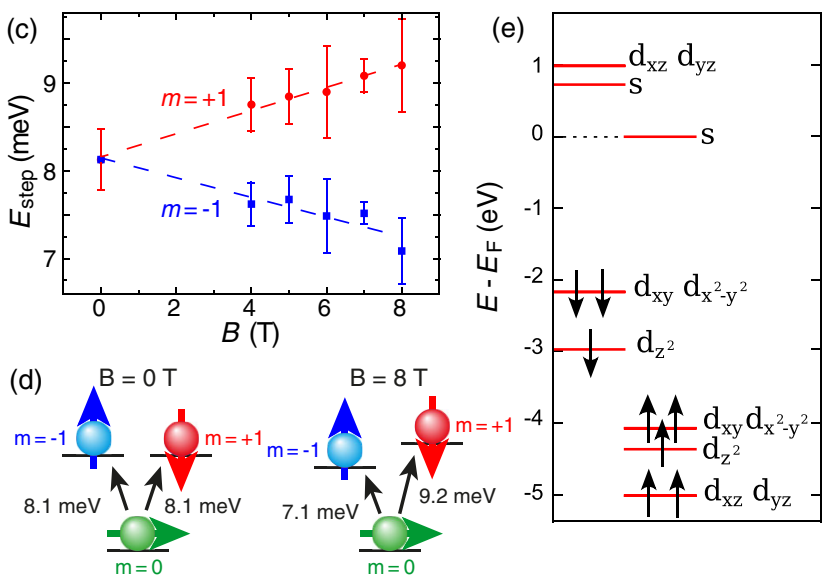

FIG. 3 (color online). (a) Field-dependent $d I / d V$ curves of a clean Co atom proving the magnetic origin of conductance steps at $\pm 8.1 \mathrm{mV}$ (dots: raw data; lines: smoothened; $V_{t}=-15 \mathrm{mV}, I_{t}=250 \mathrm{pA}, V_{\text {mod }}=200 \mu \mathrm{V}, f=287 \mathrm{~Hz}, T=0.4 \mathrm{~K}$ ). Spectra at 4 and $8 \mathrm{~T}$ are offset for clarity. (b) $d^{2} I / d V^{2}$ of left-hand conductance step in (a) showing its Zeeman splitting. (c) Field-dependent spinexcitation energies averaged over 26 adatoms (error bars show standard deviation). (d) An external out-of-plane field lifts the excited state degeneracy leading to the observed Zeeman splitting of the spin-excitation energies. (e) DFT-calculated spin-dependent energies and filling of Co $3 d$ and $4 s$ levels in the ligand field of graphene. 
case, we find that $z$ is the out-of-plane direction. The singlet $m=0$ ground state implies a positive value of $D$, and thus a hard out-of-plane axis. Fitting the fielddependent excitation energies of Fig. 3(c), we obtain $D=$ $8.1 \pm 0.4 \mathrm{meV}$ and $g=2.2 \pm 0.4$. Deviations from the free-electron $g$ factor of 2 can be rationalized in terms of orbital contributions to the total magnetic moment, which can be significant for single adatoms [36]. Assuming $S=$ 1 , we then obtain an effective spin magnetic moment of $2.2 \pm 0.4 \mu_{B}$ and a magnetic anisotropy $K=-D S_{z}^{2}=$ $-8.1 \pm 0.4 \mathrm{meV}$ [40], with the sign convention that positive values of $K$ signify easy out-of-plane magnetization axes.

For Co on freestanding graphene, our LDA $+U$ calculations show an out-of-plane hard axis, an anisotropy value of $K=-9.55 \mathrm{meV}$, and a local spin moment of $m_{S}=$ $1.84 \mu_{B}$, all three in excellent agreement with our experimental findings and with one of the three possible scenarios formerly found by DFT [15]. The local orbital moment is predicted to have a strong anisotropy with $m_{L}^{\|}=0.70 \mu_{B}$ for the in-plane and $m_{L}^{\perp}=0.02 \mu_{B}$ for the out-of-plane magnetization. Our calculations further show that the effect of the $\mathrm{Pt}(111)$ substrate on the magnetic properties of Co adatoms is very weak [32].

The absolute value of the anisotropy is within the error bar identical to the present single atom record of $K=9.3 \pm$ $1.6 \mathrm{meV}$ measured for $\mathrm{Co} / \mathrm{Pt}(111)$, where the anisotropy is mostly caused by the spin-orbit coupling of the heavy substrate atoms [13]. Since the spin-orbit coupling is very weak in graphene, the observed anisotropy is surprisingly high. Our calculations reveal that such a high value of $K$ originates from the very strong anisotropy of the orbital moment [41]. Its calculated value $m_{L}^{\|}-m_{L}^{\perp}=0.68 \mu_{B}$ is significantly larger than in the case of Co adatoms on $\operatorname{Pt}(111)$ [13]. The orbital anisotropy is mostly an effect of the strong hybridization between Co and graphene states and stems from the almost pure axial symmetry of the sixfold adsorption site, which induces a large zero-field splitting of the lowest Co states [15]. Evidence for strong hybridization between $\mathrm{Co}$ and graphene is inferred from Cothin-film-graphene interface anisotropies $[42,43]$ and from the induced magnetization found in Co-island-graphene interfaces [44].

We finally investigated the magnetic properties of $\mathrm{CoH}_{3}$, which is the only hydrogenated complex displaying spin excitations. Its conductance steps are with $30 \%$ relative height much more pronounced than the ones of clean Co with only $3 \%$ step height. Therefore, their field splitting is evident already from the $d I / d V$ raw data shown in Fig. 4(a). The striking similarities with the magnetic behavior of clean Co atoms [see Figs. 4(b) and 4(c)] allow us to perform the same analysis as above, obtaining $D=1.70 \pm 0.05 \mathrm{meV}$ and $g=2.19 \pm 0.13$.

For $\mathrm{Ni}$ atoms on graphene, hydrogenation has been calculated to transform the nonmagnetic into a magnetic
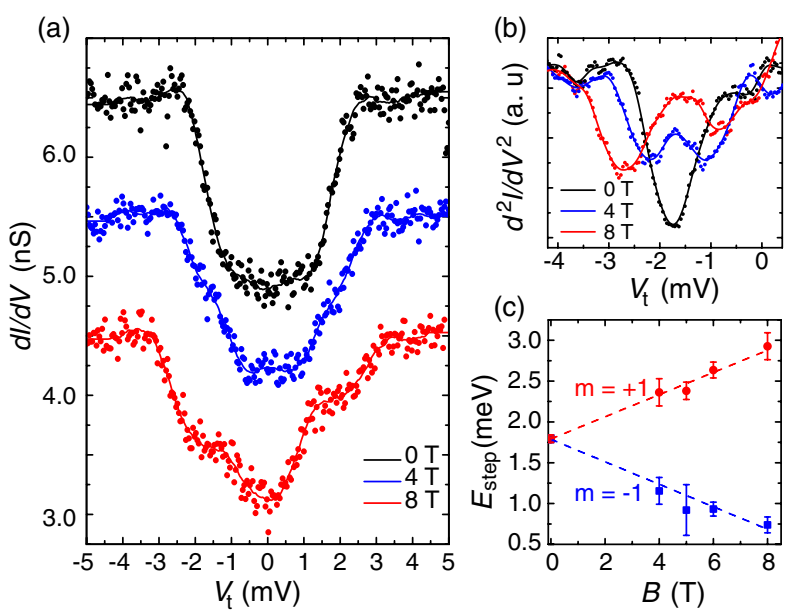

FIG. 4 (color online). (a) Field-induced splitting of the conductance steps of a $\mathrm{CoH}_{3}$ complex $\left(V_{t}=-20 \mathrm{mV}, I_{t}=\right.$ $\left.100 \mathrm{pA}, V_{\text {mod }}=200 \mu \mathrm{V}, f=611 \mathrm{~Hz}, T=0.4 \mathrm{~K}\right)$. Spectra at 4 and $8 \mathrm{~T}$ are offset for clarity. (b) Numerical derivative of the negative bias part of (a) showing each step as a minimum. Zero-field width $\Delta E=700 \pm 100 \mu \mathrm{eV}$. (c) Zeeman splitting of spin-excitation energies averaged over $15 \mathrm{CoH}_{3}$ (error bars show standard deviation).

ground state [14]. Here, we observe that the magnetic features of clean Co reappear after the adsorption of three hydrogen atoms, with identical hard axis direction, however, with significantly reduced anisotropy parameter. Since H adsorption can be made reversible by STM manipulation, one can switch between the two anisotropy values in a controlled way. However, the high reactivity of Co atoms on graphene with $\mathrm{H}_{2}$ complicates the characterization of this system with spatially integrating techniques, such as x-ray magnetic circular dichroism. We note that $\mathrm{x}$-ray magnetic circular dichroism measurements reported for Co atoms on graphene/SiC(0001) have shown very weak magnetic anisotropy, without giving access to the magnetic moment and anisotropy values [18].

In conclusion, we experimentally determined the adsorption site, magnetic ground state, and anisotropy of single Co atoms on graphene. The adsorption on the sixfold hollow site induces an unprecedentedly high anisotropy for a substrate with small spin-orbit coupling, thus rendering magnetic impurities on graphene very promising candidates for quantum magnetism and spintronics.

We acknowledge L. Claude, A. Lehnert, and M. Etzkorn for their participation in the construction of the microscope. We further acknowledge funding from the Swiss NSF under Grants No. 200020_138043, No. 200020_140479, and No. PP00P2_133552. Firstprinciples computations have been performed at the Swiss National Supercomputing Centre (CSCS) under Project No. s443. We are grateful to A. A. Khajetoorians for his fruitful comments on this manuscript. F. D. and Q.D. contributed equally to this work. 
*To whom all correspondence should be addressed. harald.brune@eplf.ch

[1] Y. W. Son, M. L. Cohen, and S. G. Louie, Nature (London) 444, 347 (2006)

[2] V. M. Karpan, G. Giovannetti, P. A. Khomyakov, M. Talanana, A.A. Starikov, M. Zwierzycki, J. vandenBrink, G. Brocks, and P. J. Kelly, Phys. Rev. Lett. 99, 176602 (2007).

[3] N. Tombros, C. Jozsa, M. Popinciuc, H. T. Jonkman, and B. J. van Wees, Nature (London) 448, 571 (2007).

[4] A. V. Krasheninnikov, P. O. Lehtinen, A. S. Foster, P. Pyykkö, and R.M. Nieminen, Phys. Rev. Lett. 102, 126807 (2009).

[5] V.W. Brar, R. Decker, H. M. Solowan, Y. Wang, L. Maserati, K. T. Chan, H. Lee, C. O. Girit, A. Zettl, S. G. Louie, M. L. Cohen, and M. F. Crommie, Nat. Phys. 7, 43 (2011).

[6] Y. Wang, V. W. Brar, A. V. Shytov, Q. Wu, W. Regan, H. Z. Tsai, A. Zettl, L.S. Levitov, and M.F. Crommie, Nat. Phys. 8, 653 (2012).

[7] H. Sevinçli, M. Topsakal, E. Durgun, and S. Ciraci, Phys. Rev. B 77, 195434 (2008).

[8] K. Sengupta and G. Baskaran, Phys. Rev. B 77, 045417 (2008).

[9] B. Uchoa, T. G. Rappoport, and A. H. Castro Neto, Phys. Rev. Lett. 106, 016801 (2011).

[10] K. M. McCreary, A. G. Swartz, W. Han, J. Fabian, and R. K. Kawakami, Phys. Rev. Lett. 109, 186604 (2012).

[11] M. Sargolzaei and F. Gudarzi, J. Appl. Phys. 110, 064303 (2011).

[12] R. J. Xiao, D. Fritsch, M. D. Kuz'min, K. Koepernik, H. Eschrig, M. Richter, K. Vietze, and G. Seifert, Phys. Rev. Lett. 103, 187201 (2009).

[13] P. Gambardella, S. Rusponi, M. Veronese, S. S. Dhesi, C. Grazioli, A. Dallmeyer, I. Cabria, R. Zeller, P.H. Dederichs, K. Kern, C. Carbone, and H. Brune, Science 300, 1130 (2003).

[14] T. O. Wehling, A. I. Lichtenstein, and M. I. Katsnelson, Phys. Rev. B 84, 235110 (2011).

[15] T.O. Wehling, A. V. Balatsky, M.I. Katsnelson, A.I. Lichtenstein, and A. Rosch, Phys. Rev. B 81, 115427 (2010).

[16] O. V. Yazyev and A. Pasquarello, Phys. Rev. B 82, 045407 (2010).

[17] A. N. Rudenko, F. J. Keil, M. I. Katsnelson, and A. I. Lichtenstein, Phys. Rev. B 86, 075422 (2012).

[18] T. Eelbo, M. Waśniowska, P. Thakur, M. Gyamfi, B. Sachs, T. O. Wehling, S. Forti, U. Starke, C. Tieg, A. I. Lichtenstein, and R. Wiesendanger, Phys. Rev. Lett. 110, 136804 (2013).

[19] J. Wintterlin and M.L. Bocquet, Surf. Sci. 603, 1841 (2009).

[20] A. J. Heinrich, J. A. Gupta, C. P. Lutz, and D. M. Eigler, Science 306, 466 (2004).

[21] H. Brune, Surf. Sci. Rep. 31, 125 (1998).

[22] V. I. Anisimov, J. Zaanen, and O. K. Andersen, Phys. Rev. B 44, 943 (1991).

[23] M. Cococcioni and S. de Gironcoli, Phys. Rev. B 71, 035105 (2005).
[24] P. Giannozzi et al., J. Phys. Condens. Matter 21, 395502 (2009).

[25] A. Dal Corso and A. Mosca Conte, Phys. Rev. B 71, 115106 (2005).

[26] J. Tersoff and D. R. Hamann, Phys. Rev. B 31, 805 (1985).

[27] M. Gao, Y. Pan, L. Huang, H. Hu, L. Z. Zhang, H. M. Guo, S. X. Du, and H. J. Gao, Appl. Phys. Lett. 98, 033101 (2011).

[28] M. Papagno, D. Pacilé, D. Topwal, P. Moras, P. M. Sheverdyaeva, F. D. Natterer, A. Lehnert, S. Rusponi, Q. Dubout, F. Calleja, E. Frantzeskakis, S. Pons, J. Fujii, I. Vobornik, M. Grioni, C. Carbone, and H. Brune, ACS Nano 6, 9299 (2012).

[29] T. A. Land, T. Michely, R. J. Behm, J. C. Hemminger, and G. Comsa, Surf. Sci. 264, 261 (1992).

[30] P. Merino, M. Švec, A. L. Pinardi, G. Otero, and J. A. Martín-Gago, ACS Nano 5, 5627 (2011).

[31] T. Eelbo, M. Waśniowska, M. Gyamfi, S. Forti, U. Starke, and R. Wiesendanger, Phys. Rev. B 87, 205443 (2013).

[32] See Supplemental Material at http://link.aps.org/ supplemental/10.1103/PhysRevLett.111.236801 for additional information about the identification and the magnetic characterization of differently hydrogenated species, the threshold tunnel voltages for transformation of one species into the other, as well as the DFT calculations of the Co adsorption sites and the influence of the Pt substrate.

[33] M. Pivetta, M. Ternes, F. Patthey, and W. D. Schneider, Phys. Rev. Lett. 99, 126104 (2007).

[34] F. D. Natterer, F. Patthey, and H. Brune, Surf. Sci. 615, 80 (2013).

[35] A. A. Khajetoorians, S. Lounis, B. Chilian, A. T. Costa, L. Zhou, D. L. Mills, J. Wiebe, and R. Wiesendanger, Phys. Rev. Lett. 106, 037205 (2011).

[36] H. Brune and P. Gambardella, Surf. Sci. 603, 1812 (2009).

[37] A. Abragam and B. Bleaney, Electron Paramagnetic Resonance of Transition Ions (Clarendon Press, Oxford, England, 1970).

[38] For the hexagonal symmetry, the first transverse anisotropy term is of sixth order [39]; therefore, it can be omitted in Eq. (1) for $S<3$.

[39] S. K. Misra, C. P. Poole, and H. A. Farach, Appl. Magn. Reson. 11, 29 (1996).

[40] For a $m=0 \rightarrow m= \pm 1$ transition, $D$ corresponds to the zero-field excitation energy and is directly measured, while $K$ depends on the choice of $S$. Therefore, the value of $K$ inferred for $S=1$ represents a lower limit for the magnetic anisotropy.

[41] P. Bruno, Phys. Rev. B 39, 865 (1989).

[42] C. Vo-Van, Z. Kassir-Bodon, H.X. Yang, J. Coraux, J. Vogel, S. Pizzini, P. Bayle-Guillemaud, M. Chshiev, L. Ranno, V. Guisset, P. David, V. Salvador, and O. Fruchart, New J. Phys. 12, 103040 (2010).

[43] N. Rougemaille, A. T. N'Diaye, J. Coraux, C. Vo-Van, O. Fruchart, and A. K. Schmid, Appl. Phys. Lett. 101, 142403 (2012).

[44] R. Decker, J. Brede, N. Atodiresei, V. Caciuc, S. Blügel, and R. Wiesendanger, Phys. Rev. B 87, 041403 (2013). 


\title{
Supplementary Material for "Magnetic Moment and Anisotropy of Individual Co Atoms on Graphene"
}

\author{
F. Donati, ${ }^{1, *}$ Q. Dubout,${ }^{1, *}$ G. Autès,${ }^{2}$ F. Patthey,${ }^{1}$ F. Calleja,,${ }^{1,3}$ P. Gambardella,,${ }^{1,4,5}$ O. V. Yazyev, ${ }^{2}$ and H. Brune ${ }^{1}$ \\ ${ }^{1}$ Institute of Condensed Matter Physics (ICMP), \\ Ecole Polytechnique Fédérale de Lausanne (EPFL), Station 3, CH-1015, Switzerland \\ ${ }^{2}$ Institute of Theoretical Physics (ITP), Ecole Polytechnique Fédérale de Lausanne (EPFL), CH-1015 Lausanne, Switzerland \\ ${ }^{3}$ Madrid Institute for Advanced Studies, IMDEA Nanoscience, \\ Calle Faraday 9, Campus Cantoblanco, E-28049 Madrid, Spain \\ ${ }^{4}$ Catalan Institute of Nanotechnology (ICN), UAB Campus, E-08193 Barcelona, Spain \\ ${ }^{5}$ Department of Materials, Eidgenössische Technische Hochschule (ETH) Zürich, CH-8093 Zürich, Switzerland.
}

\section{EXPOSURE TO RESIDUAL GAS AND TO $\mathrm{H}_{2}$}

Additional evidence for the identification of the adsorption complexes is provided by the exposure of a freshly prepared Co/graphene/Pt(111) sample to the residual gas of the UHV chamber hosting the STM cryostat, as well as to a partial pressure of $\mathrm{H}_{2}$ backfilled into this chamber. A freshly prepared sample exhibits a majority of adsorbate species D, followed by species C, B, and A, see the abundance chart in Fig. S1(a). The adsorbates were identified from their characteristic inelastic differential conductance features (see Fig. 2(c) of main paper). Exposing this sample to the residual gas pressure of the UHV chamber of $p_{\text {tot }}=7 \times 10^{-10}$ mbar by opening of the STM shutter of the cryostat for $60 \mathrm{~s}$ leads to the distribution presented in Fig. S1(b). Notice that this distribution characterizes the same adsorbates as Fig. S1(a) since we investigated the same surface spot as before the exposure. Species D has strongly diminished and the other three species, most of all $\mathrm{C}$, have increased. Note that the order of the relative abundances is still $n_{\mathrm{D}}>n_{\mathrm{C}}>n_{\mathrm{B}}>n_{\mathrm{A}}$. The sample was then exposed to $4 \times 10^{-9} \mathrm{mbar} \mathrm{H}_{2}$ for $10 \mathrm{~s}$. Figure $\mathrm{S} 1(\mathrm{c})$ presents the corresponding abundance histogram, again for the same sample location. The relative abundances of the species has reversed; now type A represents the majority, and $n_{\mathrm{D}}<n_{\mathrm{C}}<n_{\mathrm{B}}<n_{\mathrm{A}}$. Since $\mathrm{H}_{2}$-exposure creates type A and almost extinguishes type $\mathrm{D}$, and since a freshly prepared sample has mostly type $\mathrm{D}$, one identifies $\mathrm{A}$ as the most and $\mathrm{D}$ as the least hydrogenated complex. Together with the unidirectional switching by the tunnel voltage towards $\mathrm{D}$, and with the comparison between STM imaging in experiment and simulations, we unequivocally identify the adsorption complex $\mathrm{A}$ as $\mathrm{CoH}_{3}, \mathrm{~B}$ as $\mathrm{CoH}_{2}, \mathrm{C}$ as $\mathrm{CoH}$, and D as clean Co.

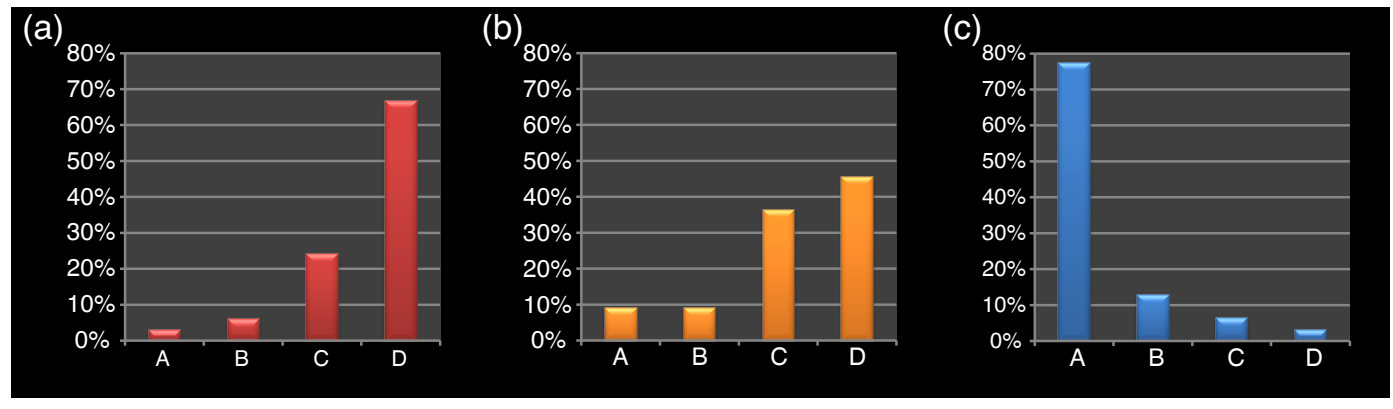

Figure S1. (a) Abundance chart of a clean sample. (b) The same adsorbates analyzed after exposure to a residual gas pressure of $p_{\text {tot }}=7 \times 10^{-10}$ mbar for $60 \mathrm{~s}$ by opening the STM shutter of the cryostat. (c) Abundance chart of the same adsorbates after exposure for $10 \mathrm{~s}$ to $p=4 \times 10^{-9}$ mbar of $\mathrm{H}_{2}$; this exposure corresponds to 0.03 Langmuir.

\section{HYDROGEN DESORPTION VOLTAGE VERSUS TUNNEL CURRENT}

We have mentioned in the main paper that $\mathrm{H}$ can be removed in an atom by atom way from $\mathrm{CoH}_{n}$ complexes since each step $n \rightarrow n-1$ has its characteristic threshold tunnel voltage. This offers the possibility to switch every $\mathrm{CoH}_{n}$ complex into any one of the less-hydrogenated ones. Here we report on an experiment that determines the respective threshold voltages and sheds light on the desorption mechanism induced by the tunnel electrons. 
Centering the tip above a $\mathrm{CoH}_{n}$ complex, and ramping the tunnel voltage with the feedback loop turned on, leads to a jump in the apparent height each time one $\mathrm{H}$ atom desorbs (for the characteristic apparent heights see Fig. 2(b) of main paper). The resulting threshold voltages are reported as function of tunnel current in Fig. S2(a) for $n=3 \rightarrow 2$ (black) and $n=2 \rightarrow 1$ (red), and in Fig. S2(b) for $n=1 \rightarrow 0$, i.e., for removal of the last $\mathrm{H}$ atom yielding clean Co (blue). At a given tunnel current, each process has its characteristic threshold voltage, the one for $n=3$ being smallest, and the one for $n=1$ largest. For all three processes, the threshold voltage decreases exponentially with increasing tunnel current until for the first two processes a plateau is reached at 150-200 pA.
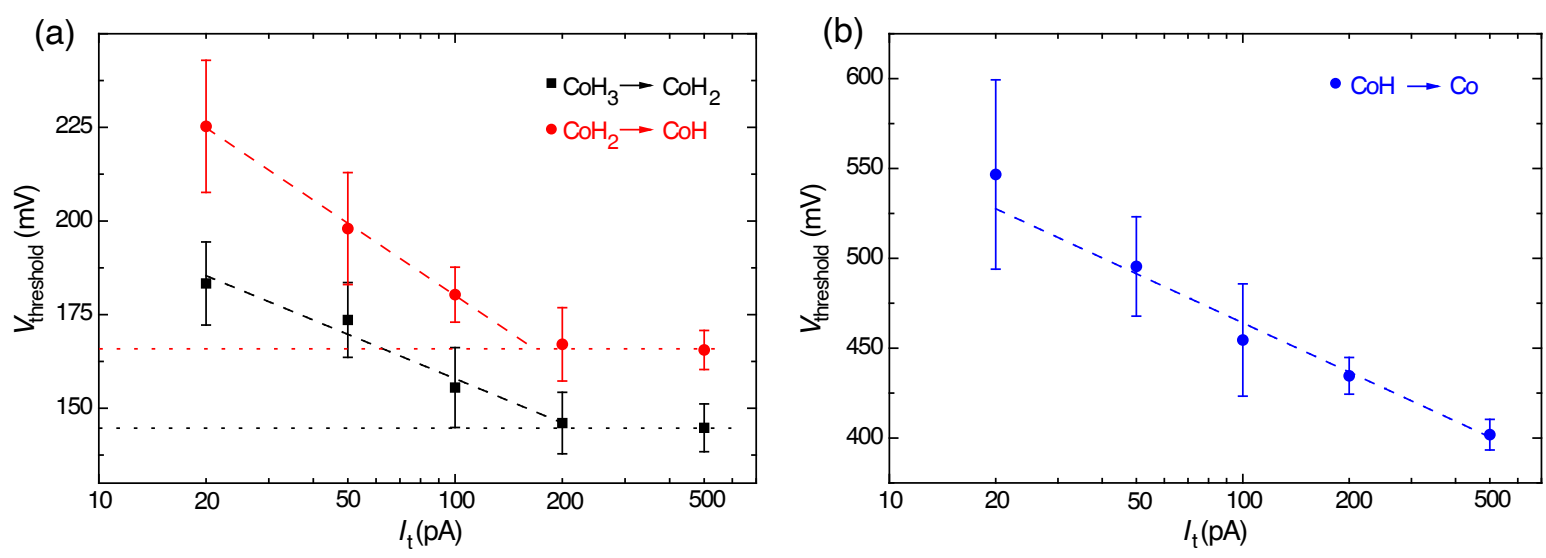

Figure S2. (a) Threshold voltages for $\mathrm{CoH}_{3} \rightarrow \mathrm{CoH}_{2}$ (black) and $\mathrm{CoH}_{2} \rightarrow \mathrm{CoH}$ (red) switching. The dashed lines are exponential fits to the first 4 points, and the dotted lines evidence the saturation at higher stabilization current (average over 5 , respectively, 20 atoms, error bars show standard deviation, $T=4.5 \mathrm{~K}$ ). (b) Threshold voltages for the CoH $\rightarrow$ Co transition. The dashed line is an exponential fit (average over 20 atoms, $T=4.5 \mathrm{~K}$ ).

In the energy range corresponding to our threshold voltages, tunnel electron stimulated desorption can either have vibrational or electronic origin [1]. In the former, vibrational levels are successively populated until the dissociation and subsequent desorption of molecular fragments occurs, while in the latter a direct transition from a bonding to an anti-bonding state produces the dissociation of the molecule and desorption of one of its constituents. Our $\mathrm{H}$ desorption threshold energies are more than an order of magnitude larger than the vibration energies detected in inelastic excitations (see Fig. 2(c) in main paper). Therefore for a vibrationally induced desorption these levels would have to be populated several times. However, desorption occurs with tunnel currents as low as $20 \mathrm{pA}$, corresponding to an average of $8 \mathrm{~ns}$ between successive tunneling electrons. From the broadening of the inelastic features in STS, the lifetime of the vibrationally excited states is with $0.1-1$ ps several orders of magnitude smaller. This excludes $\mathrm{H}$-desorption through successive vibrational excitations, suggesting instead a single-electron process in all three cases.

The observed exponential current dependence of the desorption threshold can be ascribed to electric field effects. The $x$-axes of Figs. S2 reflect the tip-sample distance, decreasing by typically $1 \AA$ per order of magnitude increase of the tunnel current. We speculate that the enhanced field at larger currents may perturb the H-Co potential landscape in such a way as to lower the energy difference between the bonding and the anti-bonding states, thus resulting in a reduced threshold voltage for desorption.

\section{ORIGIN OF THE CONDUCTANCE STEPS OF CoH AND $\mathrm{CoH}_{2}$}

In order to determine the origin of the conductance steps of $\mathrm{CoH}$ and $\mathrm{CoH}_{2}$, STS was performed on top of such complexes at $0.4 \mathrm{~K}$ with and without applying a perpendicular magnetic field of $8 \mathrm{~T}$. The corresponding $\mathrm{d} I / \mathrm{d} V$ spectra are presented in Figs. S3(a) and S4(a) respectively. These spectra were numerically differentiated in order to clearly reveal the energy position of the inelastic features, see the respective right-hand panels.

The spectrum shown in Fig. S3(a) corresponds to the average over 7 different $\mathrm{CoH}$ complexes. Three conductance steps around 2,5 and $9 \mathrm{meV}$ are visible. The precise position of these steps varies noticeably between complexes leading to the apparent peak splits in the $\mathrm{d}^{2} I / \mathrm{d} V^{2}$ curve of Fig. S3(b). Comparing the curves taken at 0 and $8 \mathrm{~T}$, some of the inelastic features apparently shift by 0.3 to $0.6 \mathrm{meV}$ with magnetic field. However, single complexes spectra (not shown) reveal that, although some features appear to shift by as much as $0.7 \mathrm{meV}$ for some complexes, the same features do not to shift at all for other complexes. Such ambiguous behavior prevents a clear identification of the 

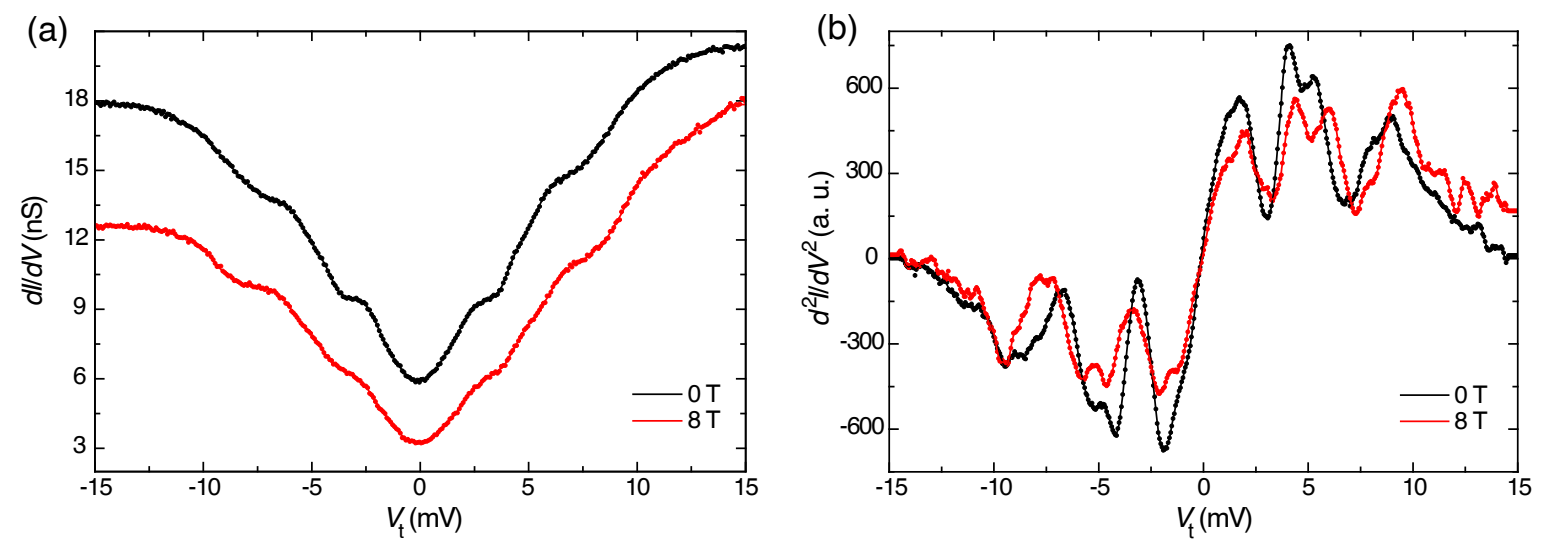

Figure S3. (a) $\mathrm{d} I / \mathrm{d} V$ spectra recorded with the tip centered above $\mathrm{CoH}$ at 0 and $8 \mathrm{~T}$. Spectrum at $8 \mathrm{~T}$ is offset for clarity. (b) The numerical derivative of (a) shows that there are no significant magnetic field effects on the excitation energies and therefore the features are associated to vibrational excitations (average over $7 \mathrm{CoH}$ complexes, stabilization of tip height at $\left.V_{\mathrm{t}}=-15 \mathrm{mV}, I_{\mathrm{t}}=200 \mathrm{pA}, V_{\bmod , \mathrm{ptp}}=300 \mu \mathrm{V}, f=611 \mathrm{~Hz}, T=0.4 \mathrm{~K}\right)$.
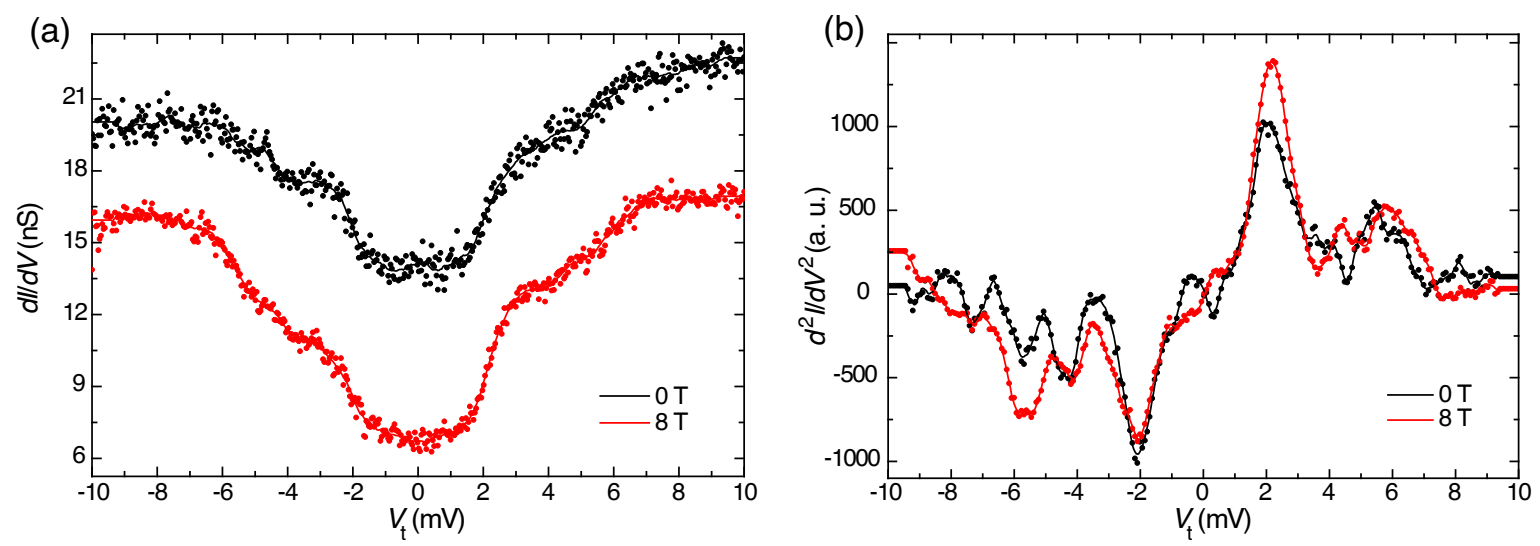

Figure S4. (a) $\mathrm{d} I / \mathrm{d} V$ spectra above $\mathrm{CoH}_{2}$ at 0 and $8 \mathrm{~T}$. Spectrum at $8 \mathrm{~T}$ is offset for clarity. (b) Numerical derivative of (a). There is no Zeeman splitting of the excitation energies identifying the excitation as vibrations ( 0 T spectrum acquired on top of a single complex, $8 \mathrm{~T}$ spectrum is average over two complexes, stabilization of tip height at $V_{\mathrm{t}}=-20 \mathrm{mV}, I_{\mathrm{t}}=100 \mathrm{pA}$, $V_{\text {mod,ptp }}=200 \mu \mathrm{V}, f=611 \mathrm{~Hz}, T=0.4 \mathrm{~K}$ ).

excitation origin. The relatively small shift values together with the absence of a clear field dependent direction of these shifts suggest a vibrational origin rather than a magnetic origin of the transition. The very subtle changes observed are most likely due to small changes of the complex orientation or configuration rather than to a spin-excitation. In the case of $\mathrm{CoH}_{2}$, two prominent features at 2 and $6 \mathrm{meV}$ can be observed and the absence of their Zeeman shift or splitting is much more evident. We therefore conclude that the observed features are of vibrational origin for both $\mathrm{CoH}$ and $\mathrm{CoH}_{2}$. We note that the absence of spin-excitations in the measured IETS spectra does not allow us to conclude that the two complexes are non-magnetic. For example, we cannot exclude that weak spin-excitations could be concealed by the large step amplitude related to the vibrational excitations. Consequently, no information can be inferred about the magnetic properties of the two complexes.

To gain insight into the nature of the vibrational excitation, a comparison between hydrogenated and deuterated Co complexes was performed. A clean sample was exposed to deuterium $\left(\mathrm{D}_{2}\right)$, obtaining the same four kind of complexes. However, no clear difference of the vibrational energies could be observed between the hydrogenated and deuterated species. This argument hints for vibrational modes involving the motion of the Co atom rather than independent motions of H/D atoms. Typical internal vibrational modes for free transition metals and rare earth hydrides are in the range of $200 \mathrm{meV}$ [2, 3], while single vertical stretching mode predicted for single Co adatoms on a $\mathrm{Cu}$ surface has an energy of $33 \mathrm{meV}$ [4]. Therefore, the low energy excitations observed in the present work cannot be ascribed to any of these modes, but more likely to frustrated translations of the whole $\mathrm{CoH}$ complex, similar to the case of 
$\mathrm{CeH} / \mathrm{Ag}(100)[5]$ and $\mathrm{CO} / \mathrm{Cu}(100)[6,7]$.

We finally note that no vibrational excitations were observed for $\mathrm{CoH}_{3}$. We speculate that, for this complex, frustrated translations could be forbidden by symmetry as a consequence of the three-fold $\mathrm{H}$ arrangement around the Co adsorbate (Fig. 2(d)), as for $\mathrm{NH}_{3}$ on fcc (111) transition metal surfaces [8].

\section{ADSORPTION SITES AND BINDING ENERGIES FROM FIRST-PRINCIPLE CALCULATIONS}

We performed first-principle calculations of the total energy for the 4 adsorbed species observed in experiment (Co, $\mathrm{CoH}, \mathrm{CoH}_{2}$ and $\left.\mathrm{CoH}_{3}\right)$ within the $\mathrm{LDA}+U$ approximation $(U=4 \mathrm{eV})$. For each adsorption complex, we compared the two most probable adsorption sites for Co: on top of a carbon atom (top) and in the center of a carbon hexagon (hex). The adatoms were placed in a $4 \times 4$ supercell of graphene and the structures were fully relaxed. From the total energies, we were able to identify the most favorable adsorptions sites and the binding energies of hydrogen on the cobalt adatoms. The binding energies were calculated as $E_{\mathrm{bind}}(n)=E_{\mathrm{CoH}_{n}}-E_{\mathrm{CoH}_{n-1}}-1 / 2 E_{\mathrm{H}_{2}}$, for $n=1,2,3$. The results are summarized in Table I.

\begin{tabular}{lcc}
$\begin{array}{l}\text { Table I. Adsorption sites and hydrogen binding energies } \\
\text { of Co atoms and } \mathrm{CoH}_{n} \text { complexes on graphene. }\end{array}$ \\
\hline \multicolumn{4}{c}{ Adsorption site } & $E_{\text {bind }}(\mathrm{eV})$ \\
\hline $\mathrm{Co}$ & hex & \\
$\mathrm{CoH}$ & top & -1.171 \\
$\mathrm{CoH}_{2}$ & top & -0.400 \\
$\mathrm{CoH}_{3}$ & hex & -0.134 \\
\hline \hline
\end{tabular}

We find that for the Co adatom and for $\mathrm{CoH}_{3}$, the adsorption site is the six-fold hollow site, while CoH and $\mathrm{CoH}_{2}$ prefer to sit on top of a carbon atom. The relaxed configurations of the hydrogenated complex are shown on Fig.S5. The binding energies reveal that the formation of a $\mathrm{CoH}_{n+1}(n=0,1,2)$ complex is always favorable in an hydrogen rich environment, in agreement with the experimental results. It should be noted that the adsorption site and magnetic configuration predicted by $\mathrm{LDA}+U$ for the Co adatom differs from previously reported results where the GGA $+U$ approximation was used and which found a top adsorption site with a $S=3 / 2$ magnetic configuration (see Refs. 14 and 15 of the main text). Nevertheless, for the $S=1$ solution, GGA $+U$ also predicts a hollow adsorption site (see Ref. 15 of the main text). The relaxed configurations have been used for simulating STM images shown in Figs. 2(d-f) of the main text.

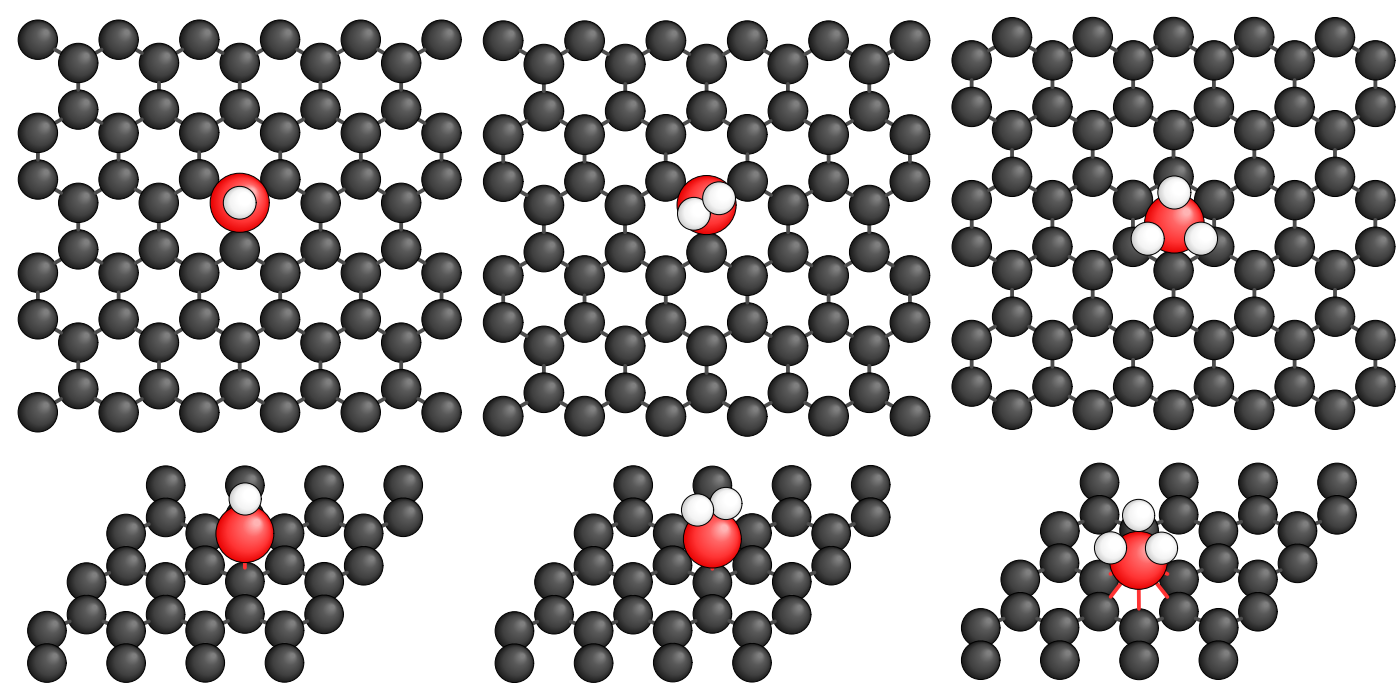

Figure S5. Top and side view of atomic structures of $\mathrm{CoH}_{n}(\mathrm{n}=1,2,3)$ complexes on graphene calculated from first principles. 


\section{INFLUENCE OF THE Pt SUBSTRATE}

Our DFT calculations described in the main text were performed using the free-standing graphene model. In order to address the role of the Pt substrate we performed a systematic study of the dependence of the magnetic properties of Co adatoms on the graphene-substrate distance. We used a $3 \times 3 \mathrm{Pt}(111)$ slab supercell with a rotated $3 \sqrt{3} \times 3 \sqrt{3} \mathrm{R} 30^{\circ}$ layer of graphene. For this configuration, the lattice mismatch between the Pt surface and the graphene layer is only $1.1 \%$. The Pt slab had a thickness of 3 monoatomic layers while the Co adatom was placed on the hexagonal site of a graphene layer. Due to the size of the unit cell, the calculations were only performed within the LDA approximation. The magnetic moment obtained with this method is smaller than the one obtained with the method LDA $+U$ used in the main text. Nevertheless, the convergence of the results with respect to the substrate/graphene distance should not depend on $U$.

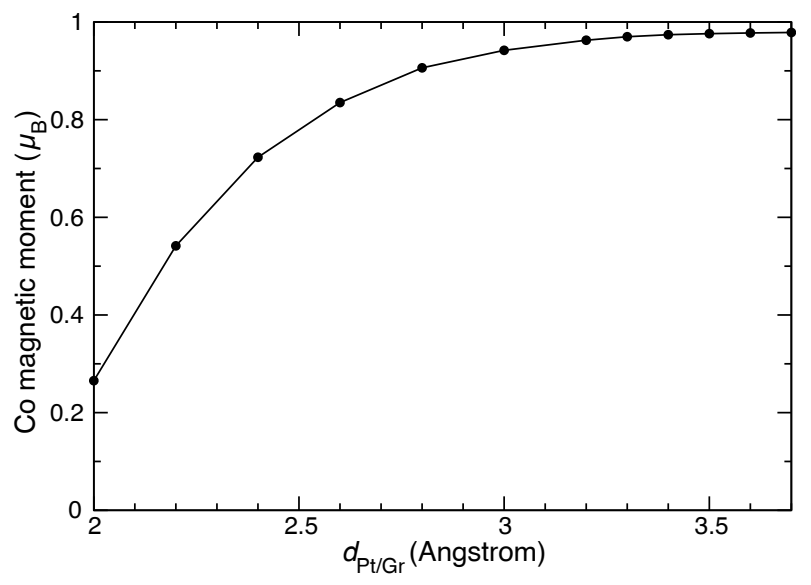

Figure S6. Calculated magnetic moment of the Co adatom as a function of the distance between the graphene layer and the Pt substrate.

Figure S6 shows the magnetic moment of the Co adatom as a function of distance between the Pt substrate and the graphene layer, $d_{\mathrm{Pt} / \mathrm{Gr}}$. We find that at small graphene-substrate distances the Co magnetic moment is strongly reduced. Experimentally reported values of $d_{\mathrm{Pt} / \mathrm{Gr}}$ range from $3.3 \AA$ [9] to $3.7 \AA$ [10]. At these distances, the magnetic moment saturates at a value of $0.98 \mu_{\mathrm{B}}$. This value is practically the same as the one obtained for a Co adatom on free-standing graphene within the same level of theory. These results demonstrate that the Pt substrate has at the experimental graphene-Pt distance no significant influence on the magnetic behavior of the Co adatoms. Therefore our experimental system is a good approximation of free-standing graphene.

* These authors contributed equally to this work.

[1] Ho, W. J. Chem. Phys. 117, 11033 (2002).

[2] Lipus, K., Bachem, E. \& Urban, W. Mol. Phys. 73, 1041 (1991).

[3] Ram, R. S. \& Bernath, P. F. J. Chem. Phys. 104, 6444 (1996).

[4] Liu, K. \& Gao, S. Phys. Rev. B 74, 195433 (2006).

[5] Pivetta, M., Ternes, M., Patthey, F. \& Schneider, W.-D. Phys. Rev. Lett. 99, 126104 (2007).

[6] Ellis, J., Toennies, J. P. \& Witte, G. J. Chem. Phys. 102, 5059 (1995).

[7] Witte, G. Surf. Sci. 502, 405 (2002).

[8] Sälli, E., Martiskainen, S. \& Martiskainen, S. J. Phys. Chem. C 116, 14960 (2012).

[9] Sutter, P., Sadowski, J. T., Sutter, E. Phys. Rev. B 80, 245411 (2009).

[10] Hu, Z.-P., Ogletree, D. F., Van Hove, M. A., Somorjai, G. A. Surf. Sci. 180, 433-459 (1987).

[11] STM images processed with WSxM (Horcas, I., Fernandez, R., Gomez-Rodriguez, J. M., Colchero, J., Gomez-Herrero, J., Baro, A. M. Rev. Sci. Instrum. 78, 013705 (2007)). 